\title{
Numerical Assesment of a Small-Scale and Very Low Tip Speed Ratio Wind Turbine
}

\author{
Martin Bourhis ${ }^{1,}$, Michaël Pereira ${ }^{1, *}$, Florent $_{\text {Ravelet }}{ }^{1, * *}$, and Ivan Dobrev ${ }^{1}$ \\ ${ }^{1}$ LIFSE, Arts et Metiers Institute of Technology, CNAM, HESAM University, 151 boulevard de l'Hopital, 75013 Paris, France
}

\begin{abstract}
The aim of this paper is to study by CFD the performance and to characterize the velocity fields in the wake of an horizontal axis wind turbine. The design of this wind turbine is far from classical as it has been designed to work at very low angular velocity to promote torque. The 8 blades are not isolated but form a high solidity blade cascade. The numerical simulation compares well to experimental data regarding the power coefficients. The analysis of the wake does show that high tangential velocities, close to the order of magnitude that was used as a design requirement, are generated and form a stable rotating wake. This rotating kinetic energy in the wake may be used to rotate a second rotor in a counter-rotating arrangment.
\end{abstract}

\section{Introduction}

Wind energy is one of the most growing source of renewable energy. This energy resource is worldwide distributed and has attracted in the past two decades a lot of research. The technology of large-scale horizontal-axis wind turbines, that is to say with rotor diameters of the order of $10 \mathrm{~m}$ is nowadays a mature technology with very high levels of performance. The best performances are obtained with three-blades horizontal-axis wind turbines, operating at tip-speed ratios $\Lambda$ of the order of 6 to 7 and that are designed with the help of the "Blade Element / Momentum theory" $[1,2]$. This kind of wind turbines can reach up to $85 \%$ of the maximum power that could be extracted from a stream tube of cross section equal to the are that is swept by the rotor - the Betz limit.

The downsizing of wind turbines towards diameters of the order of 100 to $500 \mathrm{~mm}$ may be of interest to use them in urban environment, to take advantage of natural air circulation in buildings and to power autonomous connected sensors in the field of IOT [3-6]. This downsizing is not straightforward. Foremost, theses machines operate at lower Reynolds numbers, close to the boundary layers transition and the aerodynamic profiles have to be adapted to this [7-11]. Then, when lowering the mechanical power while keeping a relatively high angular velocity $\omega$, the mechanical torque generated by the wind turbine $T$ may eventually become far too small to cope with mechanical friction in the bearings. One way of facing this problem is to lower the angular velocity, that is to design wind turbines with much smaller tip-speed ratios $\Lambda$.

Let us recall some basic concepts about horizontal-axis wind turbines. For an incoming wind of air with density $\rho$ flowing at velocity $V_{\infty}$ and for a rotor of tip radius $R_{t}$,

\footnotetext{
*e-mail: michael.pereira@ensam.eu

**e-mail: florent.ravelet@ensam.eu
}

the available power is $P_{a}=\frac{1}{2} \rho V_{\infty}^{3} \pi R_{t}^{2}$. The maximum amount of mechanical power that could be extracted from this stream tube is given by the Betz limit $P_{B}=\frac{16}{27} P_{a}$ [2]. The power coefficient $C_{p}$ is defined as the ratio of the actual mechanical power $P=T \omega$ to the available power is $P_{a}: C_{p}=\frac{P}{P_{a}}$ and thus the Betz limit corresponds to $C_{p} \leqslant \frac{16}{27} \simeq 0.59$. The tip-speed ratio is the ratio of the tip-velocity to the incoming wind velocity: $\Lambda=\frac{R_{t} \omega}{V_{\infty}}$. Formally, the Betz limit which is obtained with the axial momentum balance equation corresponds to rotors of infinite $\Lambda$ and both axial and angular momentum balances have to be taken into account. Among various models, the optimal model of "Glauert" gives for instance a maximum $C_{p} \simeq 0.54$ for $\Lambda=3$ and $C_{p} \simeq 0.42$ for $\Lambda=1$ [12].

Most of the horizontal axis wind turbines with tip radius in the range $250 \leqslant R_{t} \leqslant 500 \mathrm{~mm}$ are designed to operate at a tip-speed ratio around $\Lambda=3[3,8,10,11]$ and have been designed with the Blade Element / Momentum Theory, using 3 or 4 blades that are considered to work as independent lifting profiles. In the present study, a rotor of tip radius $R_{t}=150 \mathrm{~mm}$ is considered. The tipspeed ratio is chosen to be $\Lambda=1$. For such a low value, the blade cascade solidity $\sigma$, which is defined as the ratio of the chord length to the azimuthal pitch, would become large and the turbine may have more blades [13]; and as stated by Sharpe [1]: "Such turbines would have to be of very high solidity, with a large number of blades. To complete the design, the blade element theory would need to be employed, but the only available blade element data are for isolated, two-dimensional aerofoils. The mutual interactions between the closely spaced, probably overlapping, blades would be large and so the available aerofoil data would be useless for the purpose of designing high-solidity turbines".

We thus propose a different design method for a rotor with $R_{t}=150 \mathrm{~mm}, \Lambda=1$ and 8 blades, that is based on 
the Euler's turbomachinery theorem. The aim of this paper is to numerically assess the performance of this wind turbine, and to give insight into the wake velocity and pressure fields, with the underlying idea of getting strong rotating component in the wake that could be used to rotate a second rotor in a counter-rotating arrangment for a future work [14-17].

\section{Design of the turbine}

The design is based on the Euler's turbomachinery theorem, i.e. on the angular momentum balance, and on the classical axial momentum balance accross an actuator disk. The problem will be considered as axisymmetric. If one considers an annular streamtube between radii $r$ and $r+d r$ through which a mass flow-rate $d \dot{m}$ circulates, and if index 1 refers to upstream of the rotor and 2 downstream of it, the angular momentum balance reads:

$$
d \dot{m}\left(r C_{\theta, 2}-r C_{\theta, 1}\right)=d T
$$

where $d T$ is the elementary torque that is applied on the fluid, and $C_{\theta}$ is the tangential velocity in a Galilean reference frame. Multiplying this equation with the angular velocity of the rotor $\omega$ would give the elementary mechanical power that is transfered to the shaft. In the classical axial momentum theory, with uniform axial velocities and equal and uniform far upstream and downstream pressures, one introduces the axial induction factor $a$ which accounts for the velocity defect in the wake of the active disk in such a way that the velocity accross the disk is $(1-a) V_{\infty}$. Then the total power that is extracted from the fluid reads in dimensionless form

$$
C_{p, w}=4 a(1-a)^{2}
$$

The maximum, i.e. the Betz limit, is obtained for $a=1 / 3$. If, we make the asumption that the process is

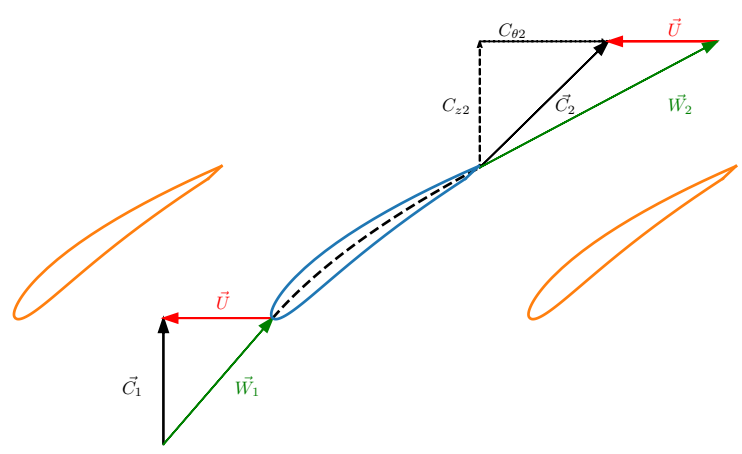

Figure 1. Blade cascade and velocity triangle at mean radius $r=\frac{R_{h}+R_{t}}{2}$. The rotation plane is horizontal. Index 1 refers to upstream, 2 to downstream of the blade cascade. $\vec{U}$ is the solid body peripheral velocity, $\vec{W}$ the relative velocity in the rotor's reference frame and $\vec{C}=\vec{U}+\vec{W}$ the absolute velocity. At this peculiar radius, the blade cascade solidity is $\sigma=0.99$

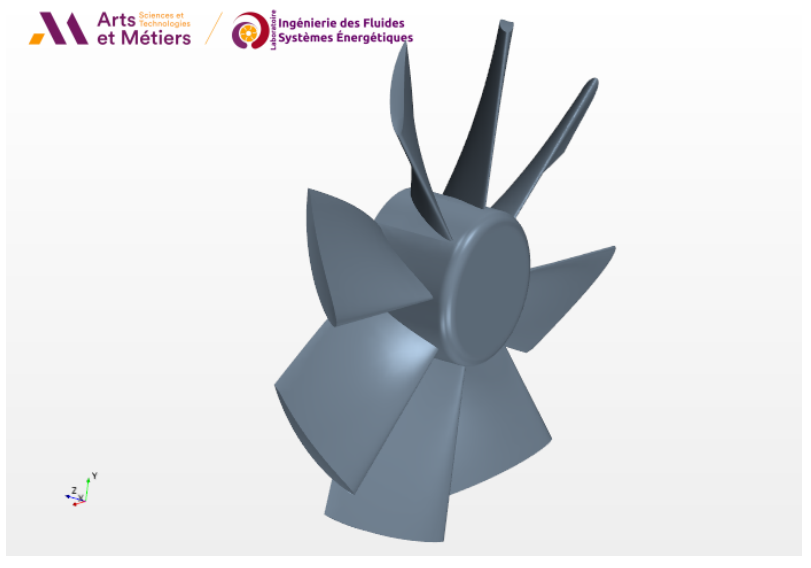

Figure 2. CAD of the horizontal axis wind turbine, based on blade cascade design

isentropic, that the discharging flow is uniform and that $C_{\theta, 1}=0$, then, one should have, with $R_{h}$ the hub radius and $\mathcal{H}=\frac{R_{h}}{R_{t}}$ the hub ratio:

$$
\begin{gathered}
\int_{R_{h}}^{R_{t}} d \dot{m} \omega\left(r C_{\theta, 2}\right)=4 a(1-a)^{2} \frac{1}{2} \rho V_{\infty}^{3} \pi R_{t}^{2} \\
\int_{R_{h}}^{R_{t}} r^{2} C_{\theta, 2} d r=a(1-a) V_{\infty}^{2} R_{t}^{2} \frac{1}{\omega}
\end{gathered}
$$

If one assumes a uniform outlet tangential velocity $C_{\theta, 2}=$ cte, it should be such that:

$$
\frac{C_{\theta, 2}}{V_{\infty}}=\frac{3 a(1-a)}{\Lambda\left(1-\mathcal{H}^{3}\right)}
$$

The main idea is to force the fluid to follow this kinematics by constraining it in a blade cascade arrangment. At each radius, a blade cascade consisting of circular lines which are tangent to the relative velocity $\vec{W}_{1}$ at leading edge and $\vec{W}_{2}$ at trailing edge is drawn (see Fig. 1). The lines are then thickened with NACA0010 profiles. The chord length of this line $c$ is determined by a choice of the local blade cascade solidity $\sigma$. With $Z$ blades, the local solidity is defined by:

$$
\sigma=\frac{Z c}{2 \pi r}
$$

According to usual axial turbines, the solidity should be of the order of 1 to ensure the required flow deviation with acceptable losses [18]. The final design of the turbine has been done with $R_{t}=150 \mathrm{~mm}, R_{h}=45 \mathrm{~mm}$, $V_{\infty}=10 \mathrm{~m} . \mathrm{s}^{-1}, \Lambda=1$-i.e. $\omega=66.7 \mathrm{rad} . \mathrm{s}^{-1}-a=1 / 3$, $Z=8$ blades and a linear variation of the local solidity from hub to tip between $\sigma_{h}=1.65$ and $\sigma_{t}=0.70$. With these parameters, the expected values of the discharging and tangential velocities in the near wake of the wind turbine are expected to be, respectively, $C_{z, 2}=0.67 V_{\infty}$ and $C_{\theta, 2}=0.68 V_{\infty}$. The resulting turbine is displayed in Fig. 2 . 


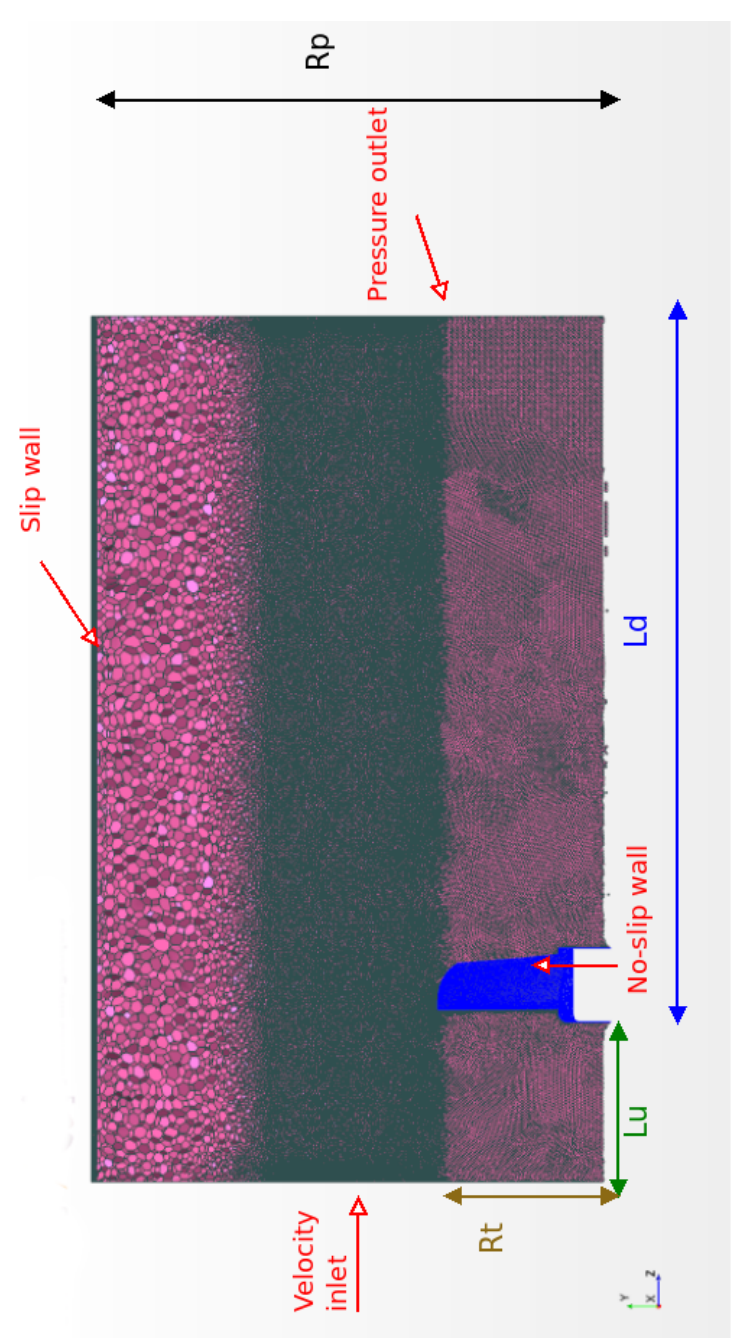

Figure 3. Typical mesh, geometric parameters and boundary conditions. $R_{t}$ is the tip radius of the turbine, $R_{p}$ the radius of the surrounding pipe, $L_{u}$ the upstream length, $L_{d}$ the downstream length

\section{Numerical setup}

The commercial Computational Fluid Dynamics code StarCCM+ 15.02 has been used to study the flow around the wind turbine. One eight of the wind turbine is simulated with periodic side boundary conditions. The simulations are steady, with a fluid of constant density and the SIMPLE pressure-velocity coupling algorithm is used. The flow is solved in a rotating reference frame. The turbulence is modeled with the $k-\omega$-SST RANS model. The mesh is polyhedral with prismatic layers close to the surface. The mesh parameters are discussed in the following, and are such that, on the one hand the dimensionless wall distance $y^{+}$are close to 3 and on the other hand the mesh is fine enough to capture the tip vortices in the wake. The quantity that is monitored is the power coefficient of the wind turbine. It is compared to experimental results obtained in the LIFSE wind tunel where the torque and angular velocity on a 3D printed wind turbine have been measured with a rotating torquemeter. In the experiment, the wind turbine is coupled to a Maxon DC motor used as a generator and loaded with a rheostat in order to explore the whole $C_{p}$ vs. $\Lambda$ characteristic curve. The torquemeter and the motor are hidden in a cylindrical pipe of diameter $42 \mathrm{~mm}$ and lenght $600 \mathrm{~mm}$ which has been modeled in the simulations as a no-slip wall, which is steady in the laboratory reference frame. In this paragraph, all the simulations are performed at $\Lambda=1$ for which the experimental power coefficient is $C_{p \text {,exp }}(1) \simeq 0.31$.

For such an external flow, the definition of the domain surrounding the wind turbine and of adequate boundary conditions is quite arbitrary. Very large domains are often used in the available litterature $[8,9,19]$. We thus first present a parametric study on the influence of the domain size and boundary conditions (see Fig. 3).
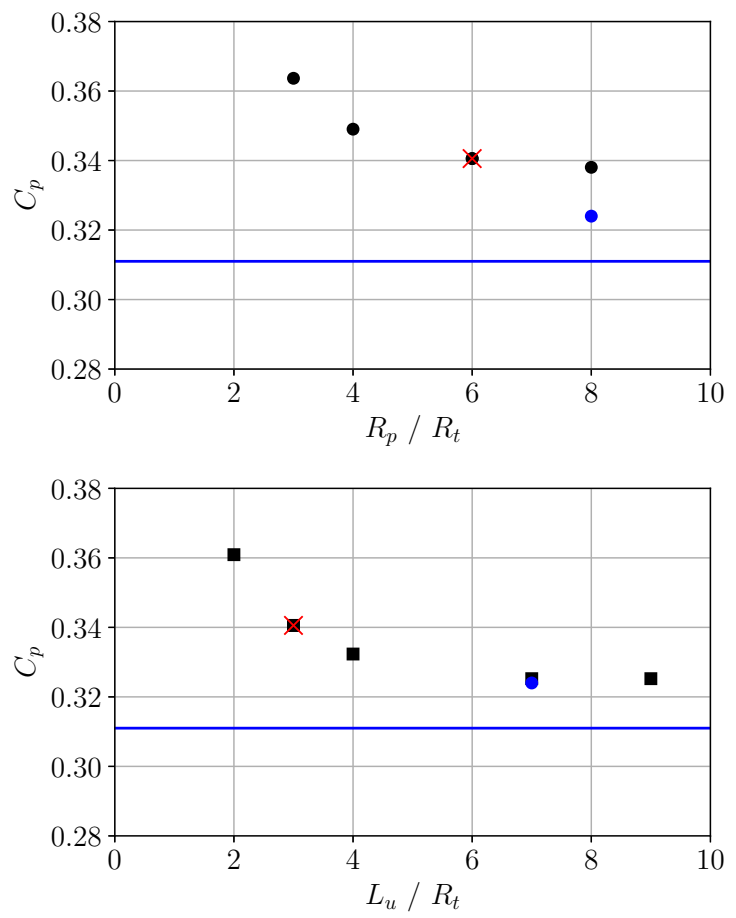

Figure 4. Effects of the domain dimensions on the power coefficient. Up: variation of $R_{p}$ at constant $L_{u}=3 R_{t}$ and $L_{d}=8 R_{t}$, bottom: variation of $L_{u}$ at constant $R_{p}=6 R_{t}$ and $L_{d}=8 R_{t}$. Black squares ( $\square)$ stand for the numerical resultswith constant meshing parameters, the blue dot $(\bullet)$ is the final configuration with a refined mesh, the solid blue line is $C_{p \text { exp }}(1)$ and the red cross $(\times)$ corresponds to the common configuration between the two plots

The inlet boundary condition consists of a uniform velocity field. The outlet boundary condition to a uniform pressure field. The upper boundary is a cylinder of radius $R_{p}$ and is a wall boundary condition. For this boundary, a comparison between slip and no-slip condition has been done and the slip conditions has been chosen as it is found to improve the estimation of the power coefficient by about $2 \%$. The length of the domain downstream of the turbine $L_{d}$ has been varied in the range 4 to $8 R_{t}$, the radius $R_{p}$ in the range 3 to $8 R_{t}$ and the upstream lenght of the domain $L_{u}$ from 2 to $9 R_{t}$.

The results are plotted in Fig; 4. All the numerical results overestimate the experimental $C_{p}$. The effect of $L_{d}$ is 
not reported as it has very lttle influence on the results in the explored range. A size of $L_{d}=8 R_{t}$ has been chosen. Surprisingly enough, the size of the upstream domain has a great influence. The convergence seems to be reached for $L_{u} \geqslant 5 R_{t}$. Concerning the surrounding pipe radius $R_{p}$, a value of $R_{p} \geqslant 6 R_{t}$ seems sufficient. Thus, the final configuration is $L_{d}=8 R_{t}, L_{u}=7 R_{t}$ and $R_{p}=8 R_{t}$.

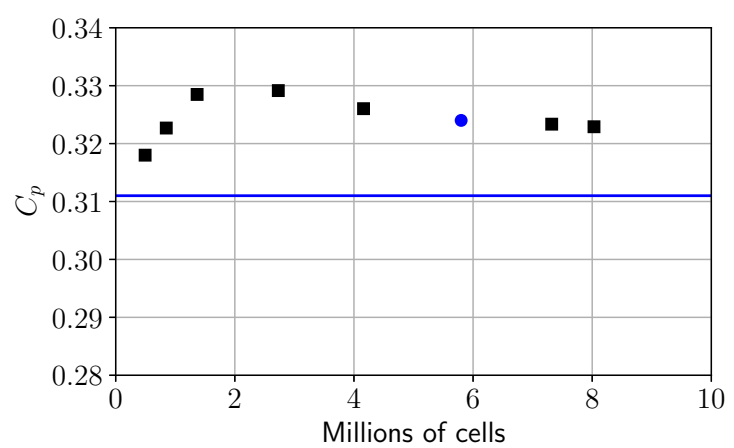

Figure 5. Grid convergence test on the final domain configuration with $L_{d}=8 R_{t}, L_{u}=7 R_{t}$ and $R_{p}=8 R_{t}$

A grid convergence test has been performed with this geometrical parameters. The base size of the mesh has been varied between $40 \mathrm{~mm}$ and $25 \mathrm{~mm}$ and the mesh has been locally refined in a cylinder domain of radius $2 R_{t}$ with a size ranging from $6 \mathrm{~mm}$ to $1.25 \mathrm{~mm}$. A prismatic layer with 8 geometrically spaced layers representing $10 \%$ of the local base size has been added around the wind turbine. The results are plotted in Fig. 5. The mesh that is considered in the following section contains 5.8 millions cells and corresponds to a base size of $30 \mathrm{~mm}$, refined to $2.25 \mathrm{~mm}$ within $r \leqslant 2 R_{t}$ and 8 geometrically spaced layers covering $3 \mathrm{~mm}$.

\section{Results and discussion}

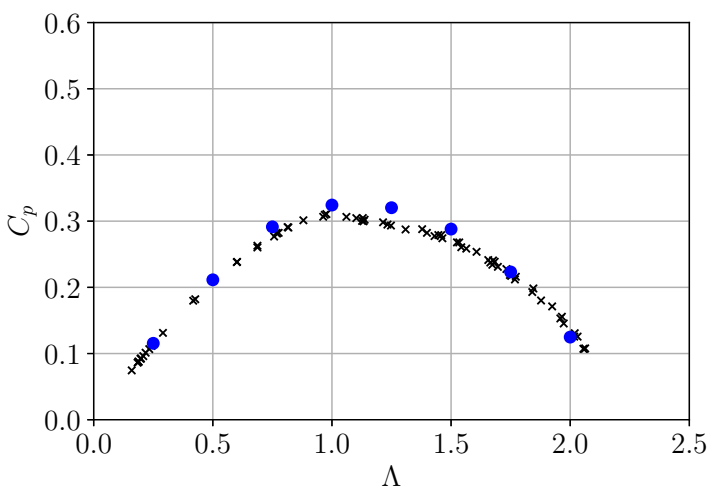

Figure 6. Power coefficient $C_{p}$ as a function of tip-speed ratio $\Lambda$. The black crosses $(\times)$ represent the experimental data and the blue dots $(\bullet)$ the results obtained with the CFD

The power coefficient $C_{p}$ for 8 values of $\Lambda$ in the range $0.25 \leqslant \Lambda \leqslant 2$ have been measured in the simulation and are reported in Fig; 6. They are compared to experimental results. The numerical results are in fairly good agreement with the experiment on the whole range of $\Lambda$. The maximum efficiency is obtained at $\Lambda=1$ which supports a first validation of the design method. The numerical result at $\Lambda=1$ is $C_{p, \text { num }} \simeq 0.324$ and overestimates the experimental one by $4 \%$.

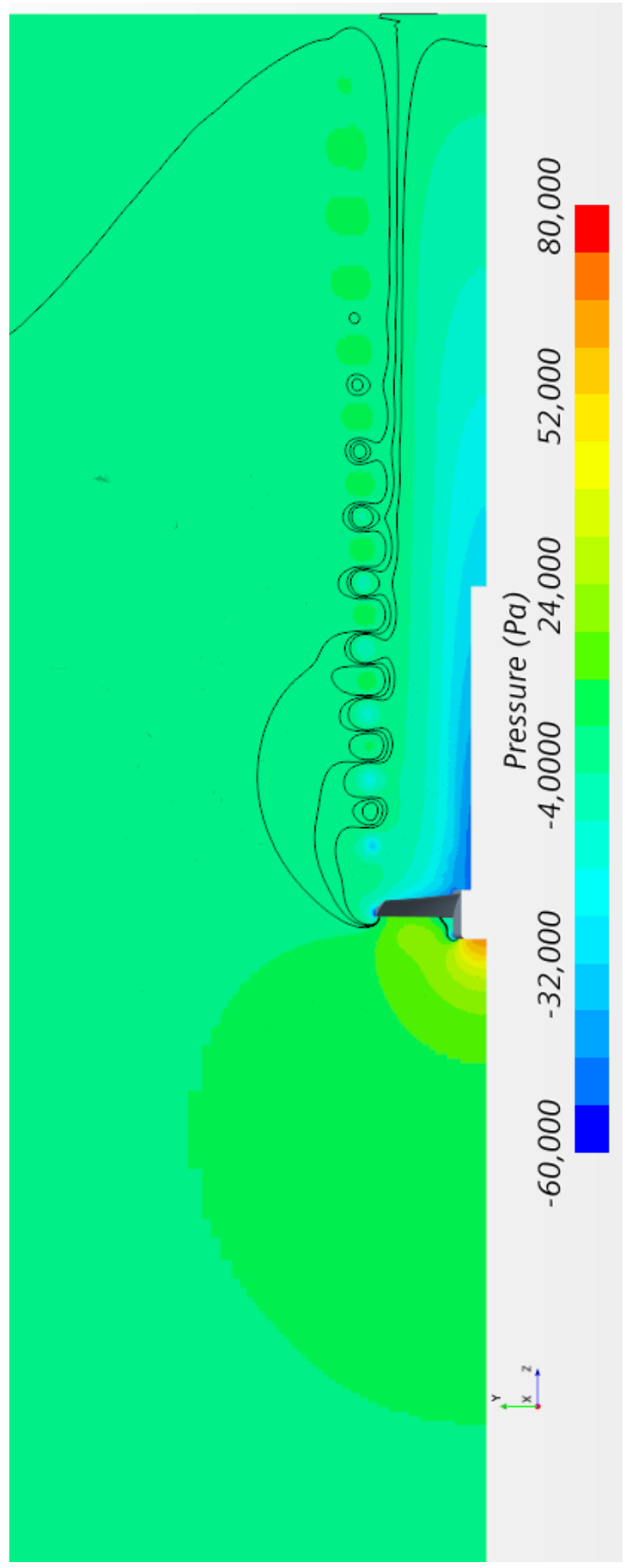

Figure 7. Static pressure field for $\Lambda=1$, with $\rho=1.2 \mathrm{~kg} \cdot \mathrm{m}^{-3}$ and $V_{\infty}=10 \mathrm{~m} \cdot \mathrm{s}^{-1}$. Mesh of 5.8 millions cells with $L_{d}=8 R_{t}$, $L_{u}=7 R_{t}$ and $R_{p}=8 R_{t}$. The black lines are iso-contours of static pressure at $-2,0$ and $+2 \mathrm{~Pa}$ 
The pressure field obtained numerically at $\Lambda=1$ in the plane $\theta=0$ that contains the leading edge of the blade is displayed in Fig. 7; the absolute tangential and axial velocity fields are displayed respectively in Fig. 8 and Fig. 9. The dynamic pressure in the incoming flow is $600 \mathrm{~Pa}$. The lowest pressure that is observed on the blades is of the order of $-60 \mathrm{~Pa}$. One can first notice a high pressure field upstream of the wind turbine in the shape of a bubble that

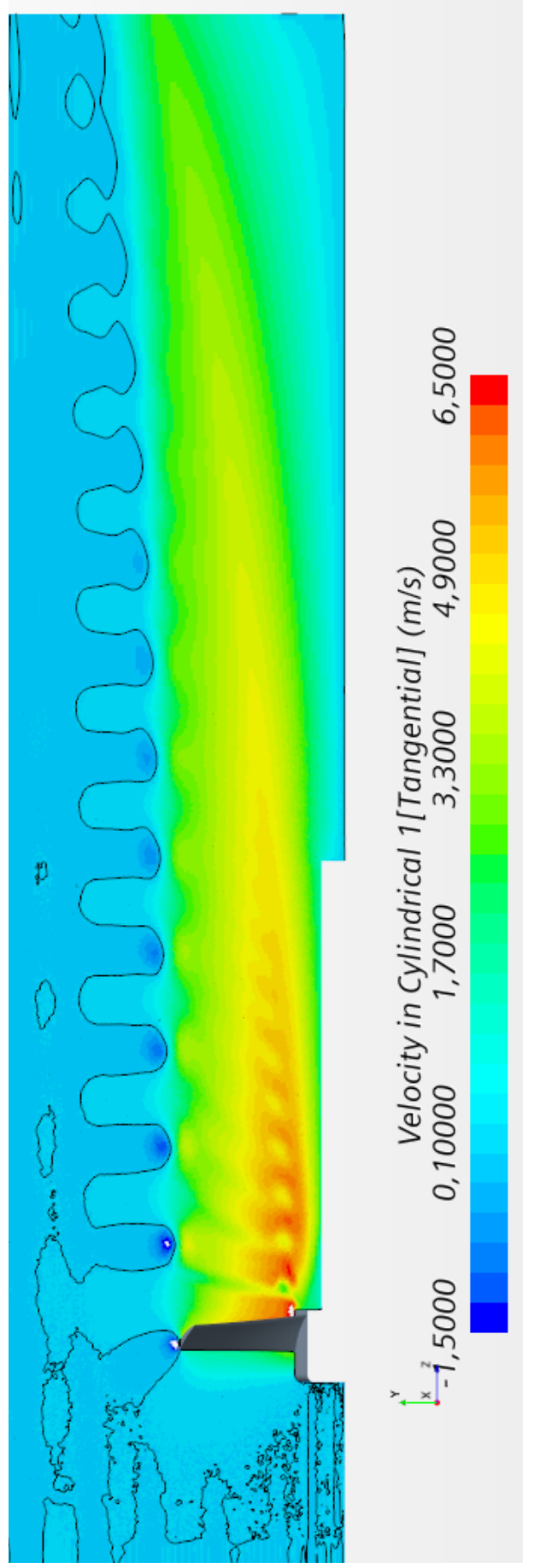

Figure 8. Absolute tangential velocity field $C_{\theta}$ for $\Lambda=1$, with $\rho=1.2 \mathrm{~kg} . \mathrm{m}^{-3}$ and $V_{\infty}=10 \mathrm{~m} . \mathrm{s}^{-1}$. Mesh of 5.8 millions cells with $L_{d}=8 R_{t}, L_{u}=7 R_{t}$ and $R_{p}=8 R_{t}$. The black line is an iso-contour of $C_{\theta}=0$ extends up to $5 R_{t}$ for $p \geqslant 4 \mathrm{~Pa}$, which may explain why an upstream length of $L_{u} \geqslant 7 R_{t}$ is necessary to reach convergence (see lower part of Fig. 4). Then, one can notice the presence of a vortex street in the wake, with a distance between the vortices of the order of $0.58 R_{t} i p$. The position of these vortices reveals the wake behind the turbine, which is diverging with an angle of about $3.5^{\circ}$.

The most noticeable feature in this wake is the presence of high tangential velocities, as expected. It is of the

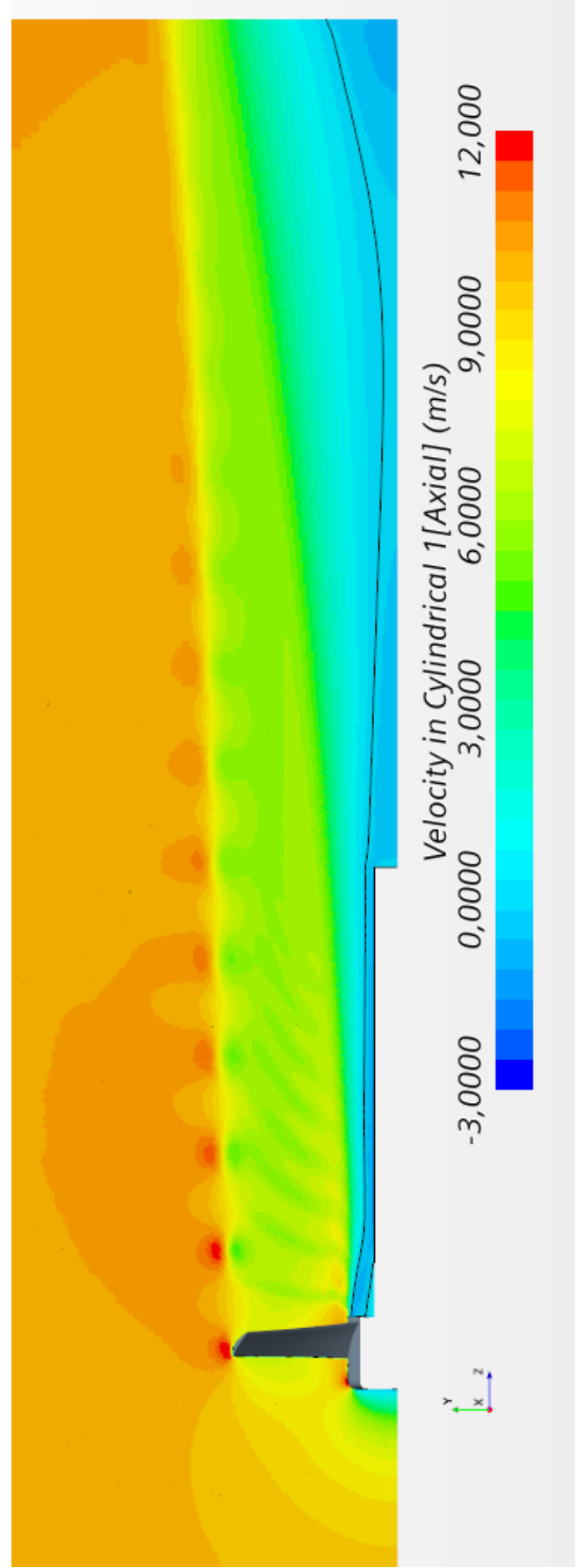

Figure 9. Absolute axial velocity field $C_{z}$ for $\Lambda=1$, with $\rho=$ $1.2 \mathrm{~kg} . \mathrm{m}^{-3}$ and $V_{\infty}=10 \mathrm{~m} . \mathrm{s}^{-1}$. Mesh of 5.8 millions cells with $L_{d}=8 R_{t}, L_{u}=7 R_{t}$ and $R_{p}=8 R_{t}$. The black line is an isocontour of $C_{z}=0$ 


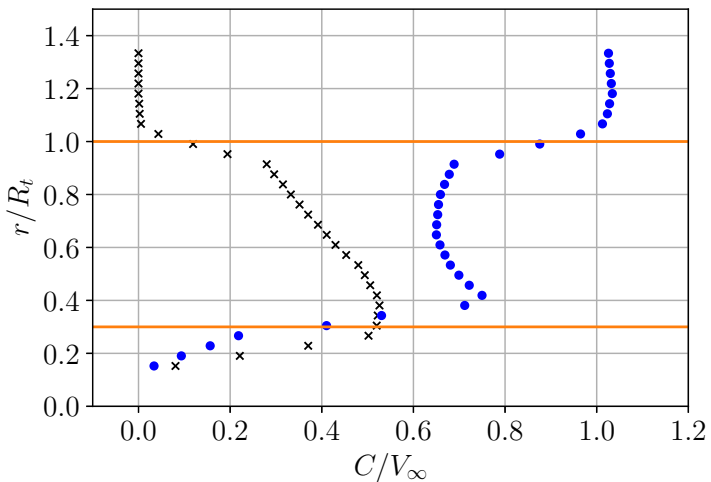

Figure 10. Radial profiles of, respectively, the azimuthallyaveraged tangential velocity $\left\langle C_{\theta}\right\rangle(r)(\times)$ and the azimuthallyaveraged axial velocity $\left\langle C_{z}\right\rangle(r)(\bullet)$, at $z=2 / 3 R_{t}$. Orange lines represent the blade hub and tip radii

order of $0.6 V_{\infty}$ and the axial velocity in the wake is of the same order of magnitude. The swirl number is thus of the order of the unity and, contrary to the numerical results presented in the chapter 6 of Ref. [12] for a Joukowsky rotor of similar size at $\Lambda=1$, there is no noticeable large recirculation bubble nor vortex breakdown in our case, which is consistent with preliminary flow visualizations in the wind tunnel. Profiles of azimuthally-averaged velocity fields have been extracted and are plotted in Fig. 10, at an axial position corresponding to $2 R_{t} / 3$ downstream of the blades' leading edges. The axial velocity in the wake is not uniform as stated in the design method though it is of the expected order of magnitude and corresponds to an average axial induction factor of 0.34 . The tangential velocity profile is not uniform; it decreases almost linearly from hub to tip. It is of the expected order of magnitude and large enough to get used to entrain a second rotor.

\section{Conclusion}

A wind-turbine of small-scale and operating at a small tip-speed ratio $\Lambda=1$ has been designed with an innovative design method. It has been tested experimentally in a wind tunnel and presents good performances. A numerical study has been made and shows that a stable and highly rotating wake is obtained, which could be used to entrain a second counter-rotating rotor that will be adapted to this flow field and that will be designed in a future work.

\section{References}

[1] D.J. Sharpe, Wind Energy 7, 177 (2004)

[2] M.O.L. Hansen, Aerodynamics of Wind Turbines, 3rd edn. (Routledge, 2015)

[3] H. Hirahara, M.Z. Hossain, M. Kawahashi, Y. Nonomura, Renewable Energy 30, 1279 (2005)

[4] R.A. Kishore, A. Marin, S. Priya, Energy Harvesting and Systems 1, 27 (2014)

[5] M.Y. Zakaria, D.A. Pereira, M.R. Hajj, Journal of Wind Engineering and Industrial Aerodynamics 147, 58 (2015)

[6] B. Pozo, J.A. Araujo, H. Zessin, L. Mateu, J.I. Garate, P. Spies, Applied Sciences 10, 6347 (2020)

[7] S. McTavish, D. Feszty, F. Nitzsche, Journal of Wind Engineering \& Industrial Aerodynamics 120, 81 (2013)

[8] M.H. Lee, Y.C. Shiah, C.J. Bai, Journal of Wind Engineering and Industrial Aerodynamics 149, 17 (2016)

[9] Y. Nishi, Y. Yamashita, T. Inagaki, Journal of Thermal Science 25, 355 (2016)

[10] K. Natarajan, R. Anand, J. Charan, T. Kanungo, Earth Environ. Sci. 268, 012159 (2019)

[11] A.M. Abdelsalam, W.A. El-Askary, M.A. Kotb, I.M. Sakr, Energy 216, 119304 (2021)

[12] J.N. Sørensen, General Momentum Theory for Horizontal Axis Wind Turbines, Vol. 4 of Research Topics in Wind Energy (Springer International Publishing, 2016)

[13] M.M. Duquette, J. Swanson, K.D. Visser, Wind Engineering pp. 299-316 (2003)

[14] R.W.Y. Habash, V. Groza, Y. Yang, C. Blouin, P. Guillemette, ISRN Mechanical Engineering 2011, 828739 (2011)

[15] Z. Wang, A. Ozbay, W. Tian, H. Hu, Energy 147, 94 (2018)

[16] M. Faisal, X. Zhao, M.H. Kang, K. You, IOP Conference Series: Materials Science and Engineering 926, 012017 (2020)

[17] X. Zhao, P. Zhou, X. Liang, S. Gao, Renewable Energy 146, 1 (2020)

[18] S.L. Dixon, C.A. Hall, in Fluid Mechanics and Thermodynamics of Turbomachinery (Sixth Edition) (Butterworth-Heinemann, 2010), pp. 53-96

[19] Y. Song, J.B. Perot, Wind Engineering 39, 299 (2015) 